\title{
The Generalized Riemann Problem of Linear Conjugation for Non-Homogeneous Polyanalytic Equations of Order $n$ in $W_{n, p}(D)$
}

\author{
Ali Seif Mshimba
}

\begin{abstract}
We consider a non-homogeneous polyanalytic partial differential equation of order $n$ in a simply-connected domain $D$ with smooth boundary $\partial D$ in the complex plane $\mathbb{C}$. Initially we transform the given equation into an equivalent system of integro-differential equations and then find the general solution of the former in $W_{n, p}(D)$. Next we pose and prove the solvability of a generalized Riemann problem of linear conjugation to the differential equation. This is effected by first reducing the Riemann problem to a corresponding one for a polyanalytic function. The latter is solved by first transforming it into $n$ classical Riemann problems of linear conjugation for $n$ holomorphic functions expressed in terms of the analytic functions which define the polyanalytic function. The solution of the classical Riemann problem is available in the literature.
\end{abstract}

Keywords: Polyanalytic functions, generalized Cauchy-Pompeiu integral operators of higher order, Riemann problem of linear conjugation

AMS subject classification: 30 G 30, 35 G 30, 35 J 40, 47 G 20

\section{Introduction}

We consider the following non-homogeneous polyanalytic partial differential equation of order $n$ in a given simply-connected and bounded domaun $D$ in the complex plane $\mathbb{C}$ :

$$
\frac{\partial^{n} w}{\partial \bar{z}^{n}}=F\left(z, w,\left\{\frac{\partial^{n+k} w}{\partial z^{m} \partial \bar{z}^{k}}\right\}\right) \quad \text { on } D
$$

where $n \in \mathbb{N}$ and $k, m \in \mathbb{N}_{0}$ are such that $m+k \leq n$ and $(0,0) \neq(m, k) \neq(0, n)$ and $\left\{\frac{\partial^{m+k} w}{\partial z^{m} \partial \bar{z}^{k}}\right\}$ stands for the set of all possible partial derivatives of $w$, with respect to $z$ or its complex conjugate $\bar{z}$, of order not exceeding $n$ and excluding $\frac{\partial^{n} w}{\partial \bar{z}^{n}}$. Equation (1) may be viewed as a complex normal form of the following system of two partial differential

A. S. A. Mshimba: Univ. of Dar es Salaam, Maths. Dept., P.O. Box 35062, Dar es Salaam, Tanzania; amshimba@mlimani.cs.udsm.ac.tz 
equations of real-valued functions $u=u(x, y)$ and $v=v(x, y)$ :

$$
\begin{aligned}
\sum_{j=0}^{n}( & \left.A_{n-j, j}^{(k)}(x, y) \frac{\partial^{n} u}{\partial x^{n-j} \partial y^{j}}+B_{n-j, j}^{(k)}(x, y) \frac{\partial^{n} v}{\partial x^{n-j} \partial y^{j}}\right) \\
& +\sum_{i+j=0}^{n-1}\left(C_{i, j}^{(k)}(x, y) \frac{\partial^{i+j} u}{\partial x^{i} \partial y^{j}}+D_{i, j}^{(k)}(x, y) \frac{\partial^{i+j} v}{\partial x^{i} \partial y^{j}}\right)=f_{k}(x, y)
\end{aligned}
$$

$(k=1,2)$. The former is obtained from the latter through the introduction of the complex notations

$$
\begin{aligned}
z=x+i y, \quad w=u+i v, \quad F & =f_{1}+i f_{2} \\
\frac{\partial}{\partial z}=\frac{1}{2}\left(\frac{\partial}{\partial x}-i \frac{\partial}{\partial y}\right) \quad \text { and } \quad \frac{\partial}{\partial \bar{z}} & =\frac{1}{2}\left(\frac{\partial}{\partial x}+i \frac{\partial}{\partial y}\right)
\end{aligned}
$$

and if the assumptions of the existence theorem on implicit functions are satisfied.

We shall show that equation (1) has a unique general solution $w$ for every prescribed in $D$ polyanalytic function of order $n$, before we embark on solving a generalized version of the Riemann problem of linear conjugation.

\section{The requisite integral operators}

When solving elliptic equations of first order (cf. [7, 15, 24, 25, 27]) the Cauchy-Pompeiu operator $T_{D}$ and the strongly singular Vekua integral operator $\Pi_{D}$ :

$$
\begin{array}{ll}
T_{D} f(z)=-\frac{1}{\pi} \iint_{D} \frac{f(\zeta)}{\zeta-z} d \xi d \eta & \\
\Pi_{D} f(z)=-\frac{1}{\pi} \iint_{D} \frac{f(\zeta)}{(\zeta-z)^{2}} d \xi d \eta & \left(\begin{array}{l}
z=x+i y \\
\zeta=\xi+i \eta
\end{array}\right)
\end{array}
$$

and the operators related to them (cf. [8, 12, 26]) play an important role. When handling elliptic differential equations of higher order, the need arises either to reduce the given equation to one of first order or, alternatively, to generalize the integral operators so that they can be applied rationally to the higher order differential equations at hand. Begehr and Hile generalized the operators as follows (see $[2,5,6]$ ):

$$
T_{m, n, D} f(z)=\iint_{D} K_{m, n}(z-\zeta) f(\zeta) d \xi d \eta \quad(m+n \geq 0)
$$

where the kernel $K_{m, n}$ of the operator is defined as follows:

$$
K_{m, n}(z)= \begin{cases}\frac{(-m) !(-1)^{m}}{(n-1) ! \pi} z^{m-1} \bar{z}^{n-1} & \text { as } 0 \geq m \in \mathbb{Z} \\ \frac{(-n) !(-1)^{n}}{(m-1) ! \pi} z^{m-1} \bar{z}^{n-1} & \text { as } 0 \geq n \in \mathbb{Z} \\ \frac{z^{m-1} \bar{z}^{n-1}}{(m-1) !(n-1) ! \pi}\left(\log |z|^{2}-\sum_{r=1}^{m-1} \frac{1}{r}-\sum_{s=1}^{n-1} \frac{1}{s}\right) & \text { as } m, n \in \mathbb{N} .\end{cases}
$$


If $m=1$ or $n=1$, the correspoding sum is dropped. The case $m=n=0$ is used to define the identity operator. Thus $T_{0,0, D} f=f$ for all $f \in L_{p}(D) \quad(1<p<\infty)$.

We note that the kernel $K_{m, n}$ has no singularity in $\mathbb{C}$, except possibly at the origin. It has at worst a pole of order two, when $m=-n$. In such a case the operator $T_{m, n, D}$ is strongly singular, and hence $T_{m, n, D} f$ exists solely in the sense of the Cauchy principal value if $f \in C_{\alpha}(D) \quad(0<\alpha<1)$ or $f \in L_{p}(D) \quad(1<p<\infty)$. As such, $T_{m, n, D}$ can be seen as a generalization of the Vekua integral operator $\Pi_{D}$. It satisfies, moreover, the Zygmund-Calderon inequality (cf. [5, 6, 9, 12, 19, 21, 26]):

$$
\begin{aligned}
& \left\|T_{m, n, D} f\right\|_{p} \leq A_{p}\|f\|_{p, D} \\
& 1=\left\|T_{m, n, D}\right\|_{2} \leq\left\|T_{m, n, D}\right\|_{p} \leq A_{p}
\end{aligned} \quad\left(\begin{array}{c}
m+n=0 \\
1<p<\infty
\end{array}\right) .
$$

If $m+n>0$, then the operator $T_{m, n, p}$ has either no singularity in $\mathbb{C}$, or it has a pole oforder 1 at $\zeta=z$. It can thus be viewed as a generalization of the Cauchy-Pompeiu operators $T_{D}, \bar{T}_{D}, T_{D}^{*}$ or the potential operator $P_{D}$ :

$$
\begin{aligned}
& \bar{T}_{D} f(z)=-\frac{1}{\pi} \iint_{D} \frac{f(\zeta)}{\bar{\zeta}-\bar{z}} d \xi d \eta \\
& T_{D}^{*} f(z)=-\frac{1}{\pi} \iint_{D} \frac{f(\zeta)}{|\zeta-z|} d \xi d \eta \\
& P_{D} f(z)=\frac{2}{\pi} \iint_{D} f(\zeta) \log |\zeta-z| d \xi d \eta .
\end{aligned}
$$

Since $K_{m, n} \in L_{1}(D)$ if $D$ has a finite area, we deduce from the convolution theorem of Young (see [19: p. 7], for instance) that $T_{m, n, D} f \in L_{p}(D) \quad(1 \leq p \leq \infty)$, and an estimate of the form

$$
\left\|T_{m, n, D} f\right\|_{p, D} \leq C(m, n, D)\|f\|_{p, D} \quad(1 \leq p \leq \infty ; m+n>0)
$$

holds.

The integral operators $T_{m, n, D}$ with $m+n>0$ and $m+n=0$ demonstrate properties which are similar to those of the operators $T_{D}$ and $\Pi_{D}$, respectively, in the Banach spaces $C_{k, \alpha}(D) \quad(0<\alpha<1)$ and $W_{k, p}(D) \quad(1<p<\infty)$ for $k \in \mathbb{N}_{0}$ (see $\left.[2,5,6]\right)$. We write here two of such properties which will play an important role in our discussion:

1. If $f \in L_{p}(D) \quad(2<p<\infty)$ and $D \subset \mathbb{C}$ is a bounded domain, then $T_{-m, n, D} f$ exists, for $m, n \in N_{0}$ with $0 \leq m<n$, in the Lebesgue sense for all $z \in \mathbb{C}$. In particular, for $z$ with $|z|<R$ the estimates

$$
\left.\begin{array}{rl}
\left|T_{-m, n, D} f(z)\right| & \leq M_{1}(m, n, p, D, R)\|f\|_{p, D} \\
\left\|T_{-m, n, D} f\right\|_{p, D} & \leq M_{2}(m, n, p, D, R)\|f\|_{p, D}
\end{array}\right\}
$$

hold.

2. If $f \in L_{p}(D) \quad(2<p<\infty)$ and $D \subset \mathbb{C}$ is a bounded domain, then $T_{-m, n, D} f$ satisfies, for any $z_{1}, z_{2} \in \mathbb{C}$ and $m, n \in \mathbb{N}_{0}$ with $0 \leq m<n$, the Hölder condition

$$
\left|T_{-m, n, D} f\left(z_{1}\right)-T_{-m, n, D} f\left(z_{2}\right)\right| \leq M_{3}(m, n, p, D)\|f\|_{p, D}\left|z_{1}-z_{2}\right|^{\beta}
$$

where

$$
\beta= \begin{cases}1 & \text { if } n-m \geq 2 \\ 1-\frac{2}{p} & \text { if } n-m=1\end{cases}
$$




\section{Transforming (1) into an integro-differential system}

We consider the given non-homogeneous polyanalytic partial differential equation (1) in a simply-connected bounded domain $D \subset \mathbb{C}$. If $T_{m, k, D}$ denotes the generalized cauchy-Pompeiu operator (2), then $\frac{\partial}{\partial z} T_{m, k, D} f(z)=T_{m-1, k, D} f(z), \frac{\partial}{\partial \bar{z}} T_{m, k, D} f(z)=$ $T_{m, k-1, D} f(z)$ and, in general,

$$
\frac{\partial^{i+j}}{\partial z^{i} \partial \bar{z}^{j}} T_{m, k, D} f(z)=T_{m-i, k-j, D} f(z)
$$

for $m, k \in \mathbb{Z}$ and $i, j \in \mathbb{N}_{0}$ with $m+k \geq i+j \geq 0$ in the Sobolev sense (see [5]).

Suppose $w$ is a given solution of equation (1). We define a function $\Phi$ as follows:

$$
\Phi(z)=w(z)-T_{0, n, D} F\left(\zeta, w(\zeta),\left\{\frac{\partial^{m+k} w}{\partial \zeta^{m} \partial \bar{\zeta}^{k}}\right\}\right)(z) .
$$

Then, by virtue of (4), we find out immediately that

$$
\begin{aligned}
\frac{\partial^{n} \Phi}{\partial \bar{z}^{n}} & =\frac{\partial^{n} w}{\partial \bar{z}^{n}}-T_{0,0, D} F\left(\zeta, w(\zeta),\left\{\frac{\partial^{m+k} w}{\partial \zeta^{m} \partial \bar{\zeta}^{k}}\right\}\right)(z) \\
& =F\left(z, w,\left\{\frac{\partial^{m+k} w}{\partial z^{m} \partial \bar{z}^{k}}\right\}\right)-F\left(z, w,\left\{\frac{\partial^{m+k} w}{\partial z^{m} \partial \bar{z}^{k}}\right\}\right) \quad(z \in D) \\
& =0
\end{aligned}
$$

in the Sobolev sense, and as such $\Phi$ is a polyanalytic function of order $n$ in $D$ (in some literature the term " $n$-analytic function" is used synonymously). We conclude, therefore, that any solution of equation (1) may be expressed in the form

$$
w(z)=\Phi(z)+T_{0, n, D} F\left(\zeta, w(\zeta),\left\{\frac{\partial^{m+k} w}{\partial \zeta^{m} \partial \bar{\zeta}^{k}}\right\}\right)(z)
$$

where $\Phi$ is a polyanalytic function of order $n$ in the domain $D$.

On differentiating (5) we obtain

$$
h_{m, k}(z):=\frac{\partial^{m+k} w}{\partial z^{m} \partial \bar{z}^{k}}=\frac{\partial^{m+k} \Phi}{\partial z^{m} \partial \bar{z}^{k}}+T_{-m, n-k, D} F\left(\zeta, w(\zeta),\left\{\frac{\partial^{r+s} w}{\partial \zeta^{r} \partial \bar{\zeta}^{s}}\right\}\right)(z) .
$$

where $n \in \mathbb{N}$ and $m, k \in \mathbb{N}_{0}$ are such that $m+k \leq n$ and $(0,0) \neq(m, k) \neq(0, k)$. We are therefore in a position to formulate the following

Theorem 1. A function $w$ is a solution of the non-homogeneous polyanalytic partial differential equation (1) of order $n$ if and only if, for a polyanalytic function $\Phi$ of order $n$ in the domain $D \subset \mathbb{C},\left\{w, h_{m, k}\right\}$ is a solution of the integro-differential system

$$
\left.\begin{array}{rl}
w(z) & =\Phi(z)+T_{0, n, D} F\left(\zeta, w(\zeta),\left\{h_{m, k}(\zeta)\right\}\right)(z) \\
h_{m, k}(z) & =\frac{\partial^{m+k} \Phi}{\partial z^{m} \partial \bar{z}^{k}}+T_{-m, n-k, D} F\left(\zeta, w(\zeta),\left\{h_{m, k}(\zeta)\right\}\right)(z)
\end{array}\right\}
$$

where $n \in \mathbb{N}$ and $m, k \in \mathbb{N}_{0}$ are such that $m+k \leq n$ and $(0,0) \neq(m, k) \neq(0, k)$. 


\section{The general solution}

We make the following assumptions on the right-hand side of the non-homogeneous polyanalytic partial differential equation (1):

(A1) $F\left(z, w,\left\{h_{m, k}\right\}\right)$ is a continuous function of its variables $z \in D, w$ and the partial derivatives of $w$ of order not exceeding $n$ and excluding $\frac{\partial^{n} w}{\partial \bar{z}^{n}}$.

(A2) There exists a tuple $\left(w^{*},\left\{h_{m, k}^{*}\right\}\right), w^{*}, h_{m, k}^{*} \in L_{p}(D) \quad(2<p<\infty)$, such that $F\left(z, w^{*},\left\{h_{m, k}^{*}\right\}\right) \in L_{p}(D)$.

(A3) $F\left(z, w,\left\{h_{m, k}\right\}\right)$ satisfies a Lipschitz condition of the form

$$
\begin{aligned}
& \left|F\left(z, w(z),\left\{h_{m, k}(z)\right\}\right)-F\left(z, \hat{w}(z),\left\{\hat{h}_{m, k}(z)\right\}\right)\right| \\
& \leq L_{1} \max \left\{|w(z)-\hat{w}(z)|, \max _{m+k<n}\left|h_{m, k}(z)-\hat{h}_{m, k}(z)\right|\right\} \\
& \quad+L_{2} \max _{m+k=n}\left|h_{m, k}(z)-\hat{h}_{m, k}(z)\right|
\end{aligned}
$$

a.e. on $D$, if $w, \hat{w}, h_{m, k}, \hat{h}_{m, k} \in L_{p}(D)$. Whereas $0<L_{2}<1$, the constant $L_{1}$ may take any positive value.

Remark. Assumptions (A1) - (A3) guarantee that $F\left(z, w,\left\{h_{m, k}\right\}\right) \in L_{p}(D)$ whenever $h_{m, k} \in L_{p}(D) \quad(2<p<\infty)$. Indded, we have

$$
\begin{aligned}
&\left\|F\left(z, w,\left\{h_{m, k}\right\}\right)\right\|_{p, D} \\
& \leq\left\|F\left(z, w^{*},\left\{h_{m, k}^{*}\right\}\right)\right\|_{p, D} \\
&+L_{1} \max \left\{\left\|w-w^{*}\right\|_{p, D}+\max _{m+k<n}\left\|h_{m, k}-h_{m, k}^{*}\right\|_{p, D}\right\} \\
&+L_{2} \max _{m+k=n}\left\|h_{m, k}-h_{m, k}^{*}\right\|_{p, D} .
\end{aligned}
$$

We next introduce the Banach space $\mathcal{L}_{p}(D) \quad(2<p<\infty)$ :

$$
\begin{aligned}
& \mathcal{L}_{p}(D)=\left\{\left(w,\left\{h_{m, k}\right\}\right) \mid\right. \\
& w, h_{m, k} \in L_{p}(D), n \in \mathbb{N}, m, k \in \mathbb{N}_{0} \text { with } m, k \leq n,(0,0) \neq(m, k) \neq(0, n) \\
& \left.\left\|\left(w,\left\{h_{m, k}\right\}\right)\right\|_{p, D}=\max _{\gamma>0}\left\{\gamma\|w\|_{p, D}, \gamma \max _{m+k<n}\left\|h_{m, k}\right\|_{p, D}, \max _{m+k=n}\left\|h_{m, k}\right\|_{p, D}\right\}\right\} .
\end{aligned}
$$

We define a mapping $P$ in $\mathcal{L}_{p}(D)$ through the right-hand-side of (5). Namely, for any tuple $\left(w,\left\{h_{m, k}\right\}\right) \in \mathcal{L}_{p}(D)$ we set

$$
P\left(w,\left\{h_{m, k}\right\}\right)=\left(W,\left\{H_{m, k}\right\}\right)
$$

with

$$
\left.\begin{array}{rl}
W(z) & =\Phi(z)+T_{0, n, D} F\left(\zeta, w(\zeta),\left\{h_{m, k}(\zeta)\right\}\right)(z) \\
H_{m, k}(z) & =\frac{\partial^{m+k} \Phi}{\partial z^{m} \partial \bar{z}^{k}}+T_{-m, n-k, D} F\left(\zeta, w(\zeta),\left\{h_{m, k}(\zeta)\right\}\right)(z)
\end{array}\right\}
$$


where $\Phi \in W_{n, p}(D)$ is a polyanalytic function of order $n$ in $D, n \in \mathbb{N}$ and $m, k \in \mathbb{N}_{0}$ are such that $m+k \leq n$ and $(0,0) \neq(m, k) \neq(0, n)$. Surely, $P$ maps the Banach space $\mathcal{L}_{p}(D)$ into itself.

Let $\left(W,\left\{H_{m, k}\right\}\right)$ and $\left(\hat{W},\left\{\hat{H}_{m, k}\right\}\right)$ be the images of $\left(w,\left\{h_{m, k}\right\}\right)$ and $\left(\hat{w},\left\{\hat{h}_{m, k}\right\}\right)$ under the mapping $P$, respectively. Then

$$
\begin{aligned}
\gamma\|W-\hat{W}\|_{p, D} \leq & \gamma\left\|T_{0, n, D}\right\|_{p}\left\|F\left(z, w,\left\{h_{m, k}\right\}\right)-F\left(z, \hat{w},\left\{\hat{h}_{m, k}\right\}\right)\right\|_{p, D} \\
\leq & \gamma\left\|T_{0, n, D}\right\|_{p} L_{1} \max \left\{\|w-\hat{w}\|_{p, D}, \max _{m+k<n}\left\|h_{m, k}-\hat{h}_{m, k}\right\|_{p, D}\right\} \\
& +\gamma\left\|T_{0, n, D}\right\| L_{2} \max _{m+k=n}\left\|h_{m, k}-\hat{h}_{m, k}\right\|_{p, D} \\
\leq & \left\|T_{0, n, D}\right\|_{p}\left(L_{1}+\gamma L_{2}\right)\left\|\left(w,\left\{h_{m, k}\right\}\right)-\left(\hat{w},\left\{\hat{h}_{m, k}\right\}\right)\right\|_{p, D} .
\end{aligned}
$$

Similarly we obtain

$$
\left.\begin{array}{rl}
\gamma\left\|H_{m, k}-\hat{H}_{m, k}\right\|_{p, D} & \leq\left\|T_{-m, n-k, D}\right\|_{p}\left(L_{1}+\gamma L_{2}\right)\left\|\left(w,\left\{h_{m, k}\right\}\right)-\left(\hat{w},\left\{\hat{h}_{m, k}\right\}\right)\right\|_{p, D} \\
\left\|H_{\alpha, \beta}-\hat{H}_{\alpha, \beta}\right\|_{p, D} & \leq\left\|T_{-\alpha, n-\beta, D}\right\|_{p}\left(\frac{1}{\gamma} L_{1}+L_{2}\right)\left\|\left(w,\left\{h_{m, k}\right\}\right)-\left(\hat{w},\left\{\hat{h}_{m, k}\right\}\right)\right\|_{p, D}
\end{array}\right\}
$$

for $0<m+k<n, \alpha+\beta=n$ and $(\alpha, \beta) \neq(0, n)$. However, we know from properties of the integral operators $T_{m, n, D}$ that

$$
\left\|T_{-m, n-k, D}\right\|_{p}= \begin{cases}C(m, k, n, D) & \text { for } 0<m+k<n \\ \left\|\Pi_{D}\right\|_{p} & \text { for } m+k=n\end{cases}
$$

where $\Pi_{D}$ is the strongly singular Vekua integral operator. We thus conclude that

$$
\begin{aligned}
&\left\|\left(W,\left\{H_{m, k}\right\}\right)-\left(\hat{W},\left\{\hat{H}_{m, k}\right\}\right)\right\|_{p, D} \\
& \leq \max \left\{\gamma\left\|T_{0, n, D}\right\|_{p}, \gamma \max _{m+k<n}\left\|T_{-m, n-k, D}\right\|_{p},\left\|\Pi_{D}\right\|_{p}\right\} \\
& \times\left(\frac{1}{\gamma} L_{1}+L_{2}\right)\left\|\left(w,\left\{h_{m, k}\right\}\right)-\left(\hat{w},\left\{\hat{h}_{m, k}\right\}\right)\right\|_{p, D}
\end{aligned}
$$

and $P$ turns out to be contractive in $\mathcal{L}_{p}(D) \quad(2<p<\infty)$ if

$$
\max \left\{\gamma\left\|T_{0, n, D}\right\|_{p}, \gamma \max _{m+k<n}\left\|T_{-m, n-k, D}\right\|_{p},\left\|\Pi_{D}\right\|_{p}\right\}\left(\frac{1}{\gamma} L_{1}+L_{2}\right)<1
$$

If estimate (9) is realizable, then by the Banach fixed point theorem $P$ has a unique fixed point $\left(w,\left\{h_{m, k}\right\}\right) \in \mathcal{L}_{p}(D) \quad(2<p<\infty)$ satisfying (6). By Theorem 1, the corresponding $w$ is the general solution of equation (1) corresponding to the prescribed in the domain $D$ polyanalytic function $\Phi \in W_{n, p}(D)$ of order $n$.

Restriction (9) can be satisfied if the constants $L_{1}, L_{2}$ and $\gamma$ are chosen suitably and the domain $D$ is sufficiently small. It is well-known that

i) $\left\|\Pi_{D}\right\|_{p} \geq\left\|\Pi_{D}\right\|_{2}=1 \quad(1<p<\infty)$

ii) $\left\|\Pi_{D}\right\|_{p}$ is a logarithmic convex function of $p \in(1, \infty)$ 
(cf. $[5,6,9,12,13,19,20,26])$.

Suppose that $L_{2}$ satisfies the inequality $L_{2}\left\|\Pi_{D}\right\|_{p}<1$ (i.e. $0<L_{2}<1$ ). We then fix the constant $\gamma$ large enough so that, for given $L_{1}$, the relation $\left(\frac{1}{\gamma} L_{1}+L_{2}\right)\left\|\Pi_{D}\right\|_{p}<1$ is also valid. Finally, since $\left\|T_{0, n, D}\right\|_{p}$ and $\left\|T_{-m, n-k, D}\right\|_{p}$ for $m+k<n$ increase with the area of the domain $D$, a proper reduction of the size of $D$ ensures the fulfilment of estimate (9), and hence the existenxe of a unique fixed point of the operator $P$ in $\mathcal{L}_{p}(D) \quad(2<p<\infty)$.

Theorem 2. Assuming the validity of assumptions (A1) - (A3) and estimate (9), the non-homogeneous polyanalytic partial differential equation (1) of order $n$ admits a unique solution $w \in W_{n, p}(D) \quad(2<p<\infty)$ given by equation (5), for every prescribed in the bounded domain $D$ polyanalytic function $\Phi \in W_{n, p}(D) \quad(2<p<\infty)$ of order $n$.

It can also be shown that the operator $\mathcal{R}$ :

$$
\mathcal{R}\left(\Phi,\left\{\frac{\partial^{m+k} \Phi}{\partial z^{m} \partial \bar{z}^{k}}\right\}\right)=\left(w,\left\{h_{m, k}\right\}\right)
$$

is a topological mapping of the set $H$ of all in the domain $D$ polyanlytic functions $\Phi \in W_{n, p}(D)$ of order $n$ onto the set $W$ of all solutions $\left(w,\left\{h_{m, k}\right\} \in \mathcal{L}_{p}(D)\right.$ of the integro-differential system (6). In the light of Theorem 1 we can thus formulate the following

Theorem 3. The operator $\mathcal{R}$ is a topological mapping of the set $H$ of all in the domain $D$ polyanalytic functions $\Phi \in W_{n, p}(D)$ of order $n$ onto the set $W$ of all solutions $w \in W_{n, p}(D)$ of the complex partial differential equation (1).

\section{The generalized Riemann problem of linear conjugation for non-homogeneous polyanalytic equations}

We now pose the following generalized Riemann problem of linear conjugation for the function $w$ :

$$
\begin{array}{ll}
\frac{\partial^{n} w}{\partial \bar{z}^{n}}=F\left(z, w,\left\{\frac{\partial^{n+k} w}{\partial z^{m} \partial \bar{z}^{k}}\right\}\right) & \text { on } D \\
{\left[\bar{z}^{q_{j}} \frac{\partial^{2 j-1} w}{\partial z^{j-1} \partial \bar{z}^{j}}\right]^{+}(t)-G_{j}(t)\left[\bar{z}^{q_{j}} \frac{\partial^{2 j-1} w}{\partial z^{j-1} \partial \bar{z}^{j}}\right]^{-}(t)=g_{j}(t)} & \text { on } \partial D \\
{\left[\frac{\partial^{2 s-2} w}{\partial z^{s-1} \partial \bar{z}^{s-1}}\right]^{+}(t)-H_{s}(t)\left[\frac{\partial^{2 s-2} w}{\partial z^{s-1} \partial \bar{z}^{s-1}}\right]^{-}(t)=f_{s}(t)} & \text { on } \partial D
\end{array}
$$

where $q_{j} \in \mathbb{Z}$,

$$
\left.\begin{array}{c}
g_{j}, G_{j} \in W_{n-2 j+1-\frac{1}{p}, p}(\partial D) \\
f_{s}, H_{s} \in W_{n-2 s+2-\frac{1}{p}, p}(\partial D)
\end{array}\right\} \quad(2<p<\infty)
$$

and

$j, s \in \mathbb{N}, n \in \mathbb{N}_{0}$ with $2 j<n+1$ and $2 s<n+2$ 
$k, m, n \in \mathbb{N}_{0}$ with $m+k \leq n$ and $(0,0) \neq(m, k) \neq(0, n)$.

We know that the general solution of equation (1) takes the form (5), where $\Phi$ is an arbitrary polyanalytic function of order $n$ in the given domain $D$. We thus first substitute the jump conditions $(\mathrm{G})$ in the general solution (5) and thus arrive at the following corresponding problem for the polyanalytic function $\Phi$ :

$$
\begin{aligned}
& {\left[\bar{z}^{q_{j}} \frac{\partial^{2 j-1} \Phi}{\partial z^{j-1} \partial \bar{z}^{j}}\right]^{+}(t)-G_{j}(t)\left[\bar{z}^{q_{j}} \frac{\partial^{2 j-1} \Phi}{\partial z^{j-1} \partial \bar{z}^{j}}\right]^{-}(t)} \\
& \quad=g_{j}(t)+G_{j}(t) \bar{t}^{q_{j}}\left[\frac{\partial^{2 j-1}}{\partial z^{j-1} \partial \bar{z}^{j}} T_{0, n, D} F\right]^{-}(t)-\bar{t}^{q_{j}}\left[\frac{\partial^{2 j-1}}{\partial z^{j-1} \partial \bar{z}^{j}} T_{0, n, D} F\right]^{+}(t) \\
& \quad=g_{j}(t)+\left(G_{j}(t)-1\right) \bar{t}^{q_{j}} T_{1-j, n-j, D} F\left(\cdot, w,\left\{h_{m, k}\right\}\right)(t)
\end{aligned}
$$

on $\partial D$, since $T_{m, k, D} F$ is a continuous function on the whole complex plane whenever $F \in L_{p}(D), 2<p<\infty$ and $m+k>0$. Similarly, we obtain from the jump conditions $(\mathrm{H})$

$$
\begin{aligned}
& {\left[\frac{\partial^{2 s-2} \Phi}{\partial z^{s-1} \partial \bar{z}^{s-1}}\right]^{+}(t)-H_{s}(t)\left[\frac{\partial^{2 s-2} \Phi}{\partial z^{s-1} \partial \bar{z}^{s-1}}\right]^{-}(t)} \\
& \quad=f_{s}(t)+\left(H_{s}(T)-1\right) T_{1-s, n-s+1, D} F\left(\cdot, w,\left\{h_{m, k}\right\}\right)(t)
\end{aligned}
$$

on $\partial D$. We deduce from the properties of the integral operators $T_{m, k, D}$ in $L_{p}(D) \quad(1<$ $p<\infty)$ that $T_{1-j, n-j, D} F \in W_{n-2 j+1, p}(D)$ and $T_{1-s, n-s+2, D} F \in W_{n-2 s+2, p}(D)$, and hence, by the trace theorem (see $[1,11,22,23]$ ),

$$
T_{1-j, n-j, D} F \in W_{n-2 j+1-\frac{1}{p}, p}(\partial D) \quad \text { and } \quad T_{1-s, n-s+2, D} F \in W_{n-2 s+2-\frac{1}{p}, p}(\partial D) .
$$

We next split the polyanalytic function $\Phi$ into two such functions, $\Phi=\Phi_{1}+\Phi_{(w, h)}$, so that the jump conditions $(\mathrm{G})^{\prime}$ and $(\mathrm{H})^{\prime}$ are written as generalized Riemann problems of linear conjugation for $\Phi_{1}$ and $\Phi_{(w, h)}$ :

$$
\left.\begin{array}{c}
{\left[\bar{z}^{q_{j}} \frac{\partial^{2 j-1} \Phi_{1}}{\partial z^{j-1} \partial \bar{z}^{j}}\right]^{+}(t)-G_{j}(t)\left[\bar{z}^{q_{j}} \frac{\partial^{2 j-1} \Phi_{1}}{\partial z^{j-1} \partial \bar{z}^{j}}\right]^{-}(t)=g_{j}(t)} \\
{\left[\frac{\partial^{2 s-2} \Phi_{1}}{\partial z^{s-1} \partial \bar{z}^{s-1}}\right]^{+}(t)-H_{s}(t)\left[\frac{\partial^{2 s-2} \Phi_{1}}{\partial z^{s-1} \partial \bar{z}^{s-1}}\right]^{-}(t)=f_{s}(t)}
\end{array}\right\}
$$

and

$$
\left.\begin{array}{c}
{\left[\bar{z}^{q_{j}} \frac{\partial^{2 j-1} \Phi_{(w, h)}}{\partial z^{j-1} \partial \bar{z}^{j}}\right]^{+}(t)-G_{j}(t)\left[\bar{z}^{q_{j}} \frac{\partial^{2 j-1} \Phi_{(w, h)}}{\partial z^{j-1} \partial \bar{z}^{j}}\right]^{-}(t)} \\
=\left(G_{j}(t)-1\right) \bar{t}^{q_{j}} T_{1-j, n-j, D} F\left(\cdot, w,\left\{h_{m, k}\right\}\right)(t) \\
{\left[\frac{\partial^{2 s-2} \Phi_{(w, h)}}{\partial z^{s-1} \partial \bar{z}^{s-1}}\right]^{+}(t)-H_{s}(t)\left[\frac{\partial^{2 s-2} \Phi_{(w, h)}}{\partial z^{s-1} \partial \bar{z}^{s-1}}\right]^{-}(t)} \\
=\left(H_{s}(T)-1\right) T_{1-s, n-s+1, D} F\left(\cdot, w,\left\{h_{m, k}\right\}\right)(t)
\end{array}\right\}
$$

on $\partial D$. Such generalized Riemann problems of linear conjugation (10) - (11) were posed and solved by the author in [17], assuming that $\left(w,\left\{h_{m, k}\right\}\right)$ is given. It was shown there 
that such a problem admits a uniquely defined in the given domain $D$ polyanalytic function $\Phi$ of order $n$, provided that sufficient point conditions on it are imposed. The solution is an element in the Sobolev space $W_{n, p}(D) \quad(2<p<\infty)$, and it satisfies estimates of the form

$$
\|\Phi\|_{p, D} \leq C_{0}\left(p, D, Q_{j}\right) \max \left\|f_{j}\right\|_{p, \partial D}
$$

and, for $1 \leq k \leq n$,

$$
\|\Phi\|_{k, p, D} \leq C_{k}\left(p, D, Q_{j}\right) \max \left\|f_{j}\right\|_{s, p, \partial D}
$$

where

$$
s=\left\{\begin{array}{l}
n+2-k-2 j-\frac{1}{p} \\
\text { or } \\
n+1-k-2 j-\frac{1}{p},
\end{array}\right.
$$

$Q_{j}$ and $f_{j}$ are the coefficients and the free terms, respectively, in the respective Riemann problem.

We next revisit the mapping $P$ defined in Section 4 , whereby the arbitrary but fixed polyanalytic function $\Phi \in W_{n, p}(D)$ is now replaced by the one constructed above. Thus, for any tuple $\left(w,\left\{h_{m, k}\right\}\right) \in \mathcal{L}_{p}(D)$ we set

$$
P\left(w,\left\{h_{m, k}\right\}\right)=\left(W,\left\{H_{m, k}\right\}\right)
$$

with

$$
\left.\begin{array}{rl}
W(z) & =\Phi_{1}(z)+\Phi_{(w, h)}(z)+T_{0, n, D} F\left(\zeta, w(\zeta),\left\{h_{m, k}(\zeta)\right\}\right)(z) \\
H_{m, k}(z) & =\frac{\partial^{m+k}}{\partial z^{m} \partial \bar{z}^{k}}\left(\Phi_{1}+\Phi_{(w, h)}\right)(z)+T_{-m, n-k, D} F\left(\zeta, w(\zeta),\left\{h_{m, k}(\zeta)\right\}\right)(z)
\end{array}\right\}
$$

where $\Phi_{1}, \Phi_{(m, k)} \in W_{n, p}(D)$ are polyanalytic functions of order $n$ in $D, n \in \mathbb{N}$ and $m, k \in \mathbb{N}_{0}$ are such that $m+k \leq n$ and $(0,0) \neq(m, k) \neq(0, n)$.

Let

$$
\left(w,\left\{h_{m, k}\right\}\right),\left(\hat{w},\left\{\hat{h}_{m, k}\right\}\right) \in \mathcal{L}_{p}(D) \quad(2<p<\infty)
$$

and let $\left(W,\left\{H_{m, k}\right\}\right),\left(\hat{W},\left\{\hat{H}_{m, k}\right\}\right)$ be their respective images under the mapping $P$. We then obtain

$$
\begin{aligned}
\gamma \| W- & \hat{W} \|_{p, D} \\
\leq & \gamma\left\|T_{0, n, D}\right\|_{p}\left\|F\left(z, w,\left\{h_{m, k}\right\}\right)-F\left(z, \hat{w},\left\{\hat{h}_{m, k}\right\}\right)\right\|_{p, D}+\gamma\left\|\Phi_{(w, h)}-\Phi_{(\hat{w}, \hat{h})}\right\|_{p, D} \\
\leq & \gamma\left(C_{1}(p, D)\left\|T_{0, n, D}\right\|_{1, p}+\left\|T_{0, n, D}\right\|_{p}\right) \\
& \times L_{1} \max \left\{\|w-\hat{w}\|_{p, D}, \max _{m+k<n}\left\|h_{m, k}-\hat{h}_{m, k}\right\|_{p, D}\right\} \\
& +\gamma\left(C_{1}(p, D)\left\|T_{0, n, D}\right\|_{1, p}+\left\|T_{0, n, D}\right\|_{p}\right) L_{2} \max _{m+k=n}\left\|h_{m, k}-\hat{h}_{m, k}\right\|_{p, D} \\
\leq & \gamma\left(C_{1}(p, D)\left\|T_{0, n, D}\right\|_{1, p}+\left\|T_{0, n, D}\right\|_{p}\right)\left(L_{1}+\gamma L_{2}\right)\left\|\left(w,\left\{h_{m, k}\right\}\right)-\left(\hat{w},\left\{\hat{h}_{m, k}\right\}\right)\right\|_{p, D} .
\end{aligned}
$$


Similarly we arrieve at

$$
\begin{aligned}
\gamma\left\|H_{m, k}-\hat{H}_{m, k}\right\|_{p, D} \leq & \left(C_{m, k}(p, D)\left\|T_{0, n, D}\right\|_{n-m-k+1, p}+\left\|T_{-m, n-k, D}\right\|_{p}\right) \\
& \times\left(L_{1}+\gamma L_{2}\right)\left\|\left(w,\left\{h_{m, k}\right\}\right)-\left(\hat{w},\left\{\hat{h}_{m, k}\right\}\right)\right\|_{p, D}
\end{aligned}
$$

and

$$
\begin{aligned}
\left\|H_{\alpha, \beta}-\hat{H}_{\alpha, \beta}\right\|_{p, D} \leq & \left(C_{n}(p, D)\left\|T_{0, n, D}\right\|_{1, p}+\left\|T_{-\alpha, n-\beta, D}\right\|_{p}\right) \\
& \times\left(\frac{1}{\gamma} L_{1}+L_{2}\right)\left\|\left(w,\left\{h_{m, k}\right\}\right)-\left(\hat{w},\left\{\hat{h}_{m, k}\right\}\right)\right\|_{p, D}
\end{aligned}
$$

for $0<m+k<n, \alpha+\beta=n$ and $(\alpha, \beta) \neq(0, n)$. Consequently,

$$
\left\|\left(W,\left\{H_{m, k}\right\}\right)-\left(\hat{W},\left\{\hat{H}_{m, k}\right\}\right)\right\| \leq \kappa\left\|\left(w,\left\{h_{m, k}\right\}\right)-\left(\hat{w},\left\{\hat{h}_{m, k}\right\}\right)\right\|
$$

where

$$
\kappa=\left(\frac{1}{\gamma} L_{1}+L_{2}\right) \max \left\{\begin{array}{l}
\gamma C_{1}(p, D)\left\|T_{0, n, D}\right\|_{1, p}+\left\|T_{0, n, D}\right\|_{p} \\
\gamma \max _{m+k<n}\left\{C_{m, k}(p, D)\left\|T_{0, n, D}\right\|_{n-m-k+1, p}+\left\|T_{-m, n-k, D}\right\|_{p}\right\} \\
C_{n}(p, D)\left\|T_{0, n, D}\right\|_{1, p}+\left\|\Pi_{D}\right\|_{p}
\end{array}\right.
$$

The mapping $P$ is thus contractive in $\mathcal{L}_{p}(D)$ if $\kappa<1$, and in this case it has a unique fixed point $\left(w,\left\{h_{m, k}\right\}\right) \in \mathcal{L}_{p}(D) \quad(2<p<\infty)$, thanks to the Banach fixed point theorem. By Theorem 1 and the construction of the polyanalytic functions $\Phi_{1}$ and $\Phi_{(w, h)}, w$ of the fixed point $\left(w,\left\{h_{m, k}\right\}\right)$ solves the generalized Riemann problem of linear conjugation $(1),(\mathrm{G}),(\mathrm{H})$.

The contractiveness of $P$ can be arrived at in essentially the same manner as the one discussed earlier in the case of a general solution. It is noteworthy in this exercise that all constants $C_{1}(p, D), C_{m, k}(p, D)$ and $C_{n}(p, D)$ increase with the size of the domain $D$.

Theorem 4. If assumptions (A1) - (A3) are satisfied and a requisite number of point conditions are given, then the generalized Riemann problem of linear conjugation (1), (G), (H) admits a unique solution $w \in W_{n, p}(D) \quad(2<p<\infty)$.

Acknowledgement. This work was carried out during the author's visit to the Free University of Berlin (Germany) under the DAAD sponsorship in Spring 1998. The author wishes herewith to express his sincere thanks to DAAD and his host, Prof. H. Begehr of the I. Mathematics Institute, for the support, inspiration and encouragement. 


\section{References}

[1] Adams, R. A.: Sobolev Spaces. New York: Acad. Press 1975.

[2] Akal, M. S.: Boundary Value Problems for Complex Elliptic Partial Differential Equations of Higher Order. Thesis. Berlin: Free Univ. 1996, and Aachen: Shaker-Verlag 1996.

[3] Balk, M. B.: Polyanalytic Functions. Berlin: Akademie-Verlag 1991.

[4] Begehr, H.: Complex Analytic Methods for Partial Differential Equations. Singapore: World Sci. Publ. 1994.

[5] Begehr, H. and G. N. Hile: A hierarchy of integral operators. Rocky Mount. J. Math. 27 (1997), $669-706$.

[6] Begehr, H. and G. N. Hile: Higher order Cauchy-Pompeiu operator. In: Proc. Oper. Theory Compl. Hypercompl. Anal., Mexico City 1994 (eds.: E. Ramires de Arellano et al.). Providence (R.I.): Amer. Math. Soc. 1998, pp. $41-49$.

[7] Begehr, H. and G. C. Wen: Nonlinear Elliptic Boundary Value Problems and Their Applications. Harlow: Longman 1996.

[8] Bliev, N. K.: Generelized Analytic Functions in Fractional Spaces. Harlow: Longman 1997.

[9] Calderon, A. P. and A. Zygmund: On the existence of singular integrals. Acta Math. 88 (1957), $85-139$.

[10] Gakhov, F. D.: Boundary Value Problems. Oxford: Pergamon Press 1966.

[11] Kufner, A., John, O. and S. Fučik: Function Spaces. Leyden: Noordhoff Int. Publ. 1977.

[12] Monakhov, V. N.: Boundary Value Problems. New Jersey: Amer. Math. Soc. 1983.

[13] Mshimba, A. S. A.: On the $L_{p}$-norms of some singular integral operators. Afrika Math. $\mathrm{V}(1983), 34-46$.

[14] Mshimba, A. S. A.: On the Riemann boundary value problem for holomorphic functions in Sobolev spaves $W_{1, p}(G)$. Compl. Var. 14 (1990), $237-242$.

[15] Mshimba, A. S. A.: The Riemann boundary value problem for nonlinear elliptic systems in the plane in Sobolev spaves $W_{1, p}(G)$. Compl. Var. 14 (1990), $243-249$.

[16] Mshimba, A. S. A.: The Cauchy-Lebesgue integral and boundary value problems. Compl. Var. 16 (1991), $307-313$.

[17] Mshimba, A. S. A.: The generalized Riemann problem of linear conjugation for polyanalytic functions of order $n$ in $W_{n, p}(D)$. Z. Anal. Anw. 20 (2001), $505-512$.

[18] Muskhelishvili, N. I.: Singuläre Integralgleichungen. Berlin: Akademie-Verlag 1965.

[19] Neri, U.: Singular Integrals. Berlin et al.: Springer-Verlag 1971.

[20] Riesz, M.: Sur les maxima des formes bilinéares et sur les fonctionelles linèares. Acta Math. 49 (1928), $465-497$.

[21] Stein, E. M.: Singular Integrals and Differentiability of Functions. Princeton: Univ. Press 1970.

[22] Triebel, H.: Interpolation Theory, Function Spaces, Differential Operators. Amsterdam: North Holland Publ. Comp. 1978.

[23] Triebel, H.: Theory of Function Spaces. Vol. I. Basel: Birkhäuser Verlag 1983.

[24] Tutschke, W.: Die neuen Methoden der Komplexen Analysis und ihre Anwendungen auf nichtlineare Differentialgleichungssysteme (Sitzungsber. Akad. Wiss. DDR: Vol. 17N). Berlin: Dt. Verlag Wiss. 1976. 


\section{$524 \quad$ Ali Seif Mshimba}

[25] Tutschke, W.: Reduction of the problem of linear conjugation for first order nonlinear elliptic systems in the plane to an analogous problem for holomorphic function. In: Proc. Anal. Funct., Kozubnik 1979 (ed.: J. Ławrynowicz). Berlin et al.: Springer-Verlag 1980, pp. $445-455$.

[26] Vekua, I. N.: Generalized Analytic Functions. Oxford: Pergamon Press 1962.

[27] Wen, G. C. and H. Begehr: Boundary Value Problems for Elliptic Equations and Systems. Harlow: Longman 1990.

[28] Xu, Z. Y.: Nonlinear Poincaré problem for a system of first order elliptic equations in the plane. Compl. Var. 7 (1987), $363-381$.

Received 27.10.1998; in revised form 20.03.2000 